\title{
La institucionalización de la igualdad de género en Galicia: un camino abierto
}

\author{
Isabel Diz Otero \\ Universidad de Santiago de Compostela \\ isabel.diz@usc.es \\ Marta Lois González \\ Universidad de Santiago de Compostela \\ martairene.lois@usc.es
}

\begin{abstract}
Resumen
El presente artículo tiene por objetivo fundamental analizar el estado de implementación de las políticas de igualdad de género en el ámbito de la Administración pública gallega. Para ello, en primer lugar, se llevará a cabo una breve presentación de los principales conceptos y estrategias relativos al género y al feminismo institucional para, en segundo lugar, a la luz de los mismos, analizar y dar cuenta del proceso de creación y desarrollo de las políticas de igualdad de género en Galicia. En este sentido, se busca responder a las siguientes preguntas: ¿qué tipo de actuaciones en materia de igualdad se han llevado a cabo en la Comunidad Autónoma gallega?, ¿qué cambios intraorganizativos y nuevas instituciones ha supuesto la incorporación de la perspectiva de género en este nivel de gobierno?, ¿se puede hablar de madurez y consolidación del impulso institucional gallego en materia de género?', ¿qué grado de influencia han ejercido las políticas y organismos de igualdad de esta comunidad en el proceso de toma de decisiones?, ¿ha influido el color político en los cambios y la evolución de las políticas de género en Galicia?, ¿son los recursos institucionales gallegos para la igualdad del presente una garantía de futuro?
\end{abstract}

Palabras clave Igualdad de trato, acciones positivas, mainstreaming, políticas públicas de género, cuotas, organismos de igualdad, Galicia.

\section{The institutionalization of gender equality in Galicia: an open path}

\footnotetext{
Abstract

This article focuses on the implementation level of gender equality policies in the Galician public administration. With this goal, the main concepts and strategies related to gender and institutional feminism will be briefly discussed, setting the basis for addressing afterwards how gender equality policies have been set up in Galicia. Such an analysis intends to answer the following questions: what kind of actions in the field of equality has been set up within the Galician Autonomous Community?, Which organizational changes and new institutions stem from the adoption of a gender perspective in this level of government?, Is it possible to consider Galician institutional efforts in gender policies as mature and well-established?, Which degree of influence have policies and equality bodies in this Community had in the decision-making process?, Has political ideology influenced any changes in and the evolution of gender policies in Galicia?, Are current Galician institutional resources for equality a guarantee for the future?
} 
La igualdad de género ha ocupado un lugar central en la agenda política de las últimas décadas. La acción de los poderes públicos con el fin de lograr la igualdad real entre mujeres y hombres estuvo caracterizada hasta hace algunos años por la puesta en marcha de programas y acciones específicas dirigidas a las mujeres; en la actualidad, por el contrario, estas acciones han ido acompañadas de nuevos modelos de intervención que sitúan la igualdad de género en el centro de la planificación y gestión de la totalidad de las políticas públicas. Desde finales de la década de los noventa, y de manera similar a lo que ha ocurrido en los países del ámbito europeo y en España, las políticas de género en el nivel autonómico han ido incorporando nuevas estrategias de género (Bustelo, 2004; Lombardo, 2004). Este nivel es particularmente relevante por la importancia que las políticas autonómicas adquieren en un contexto de fuerte descentralización como el caso español. Esto ha implicado, entre otros elementos, la descentralización de las políticas de género y la creación de organismos específicos de promoción de la igualdad.

El presente artículo tiene por objetivo fundamental analizar el estado de implementación de las políticas de igualdad género en el ámbito de la Administración pública gallega. Para ello, en primer lugar, se llevará a cabo una breve presentación de los principales conceptos y estrategias relativos al género y al feminismo institucional para, en segundo lugar, a la luz de los mismos, analizar y dar cuenta del proceso de creación y desarrollo de las políticas de igualdad de género en Galicia. En este sentido, se busca responder a las siguientes preguntas: ¿qué tipo de actuaciones en materia de igualdad se han Ilevado a cabo en la Comunidad Autónoma gallega?, ¿qué cambios intraorganizativos y nuevas instituciones ha supuesto la incorporación de la perspectiva de género en este nivel de gobierno?, ¿se puede hablar de madurez y consolidación del impulso institucional gallego en materia de género?, ¿qué grado de influencia han ejercido las políticas y organismos de igualdad de esta comunidad en el proceso de toma de decisiones?, ¿ha influido el color político en los cambios y evolución de las políticas de género en Galicia?, ¿son los recursos institucionales para la igualdad del presente una garantía de futuro en Galicia?

\section{LA APARICIÓN DEL FEMINISMO INSTITUCIONAL}

En las últimas décadas se ha experimentado una importante implicación de los poderes públicos en la lucha contra la desigualdad de género. Los precedentes de estas actuaciones se deben buscar en las luchas de los movimientos de mujeres de la denominada "segunda ola" del feminismo que tuvieron lugar durante los años sesenta y setenta. Como consecuencia de este movimiento feminista se estableció cierto consenso político acerca de la legitimidad de las demandas de las mujeres y el reconocimiento de que los poderes públicos pueden y deben hacer algo para eliminar las

146 desigualdades de género y la discriminación de las mujeres. Si bien no existió acuerdo 
sobre cuál debe ser el papel del Estado en este proceso (Lovenduski, 1997) este movimiento va a propiciar la creación del denominado feminismo institucional o de Estado. En el marco de las Administraciones públicas, desde el momento que se asume que resulta imposible evitar el Estado, ya que está presente en la mayoría de las áreas importantes de la vida social, prolifera la creación de instituciones cuyo principal cometido es la formulación y puesta en práctica de medidas para alcanzar un mayor grado de igualdad entre mujeres y hombres. Es decir, se produce la institucionalización del feminismo en agencias u organismos para la igualdad (Stetson y Mazur, 1995).

Como en la mayoría de los países occidentales, aunque con mayor tardanza, en nuestro país se crean organismos de igualdad a partir de los años ochenta tanto a nivel estatal como a nivel autonómico. La creación en 1983 del Instituto de la Mujer significó el arranque del feminismo de Estado en España que no sólo ha tenido un impacto significativo en el planteamiento de las políticas públicas de igualdad, sino que, además, se ha convertido en el principal promotor de instituciones de este tipo, fundamentalmente, en el ámbito autonómico.

Así, a partir de 1988, año en que se crean los institutos de Andalucía y el País Vasco, se va desarrollando una red de estructuras administrativas análogas al Instituto de la Mujer en las diferentes comunidades autónomas'.

\section{LA EVOLUCIÓN DE LAS POLÍTICAS PÚBLICAS DE IGUALDAD DE GÉNERO}

Las políticas públicas de género se pueden definir, tal y como plantea Lombardo, como el "conjunto de decisiones, objetivos y medidas adoptadas por las instituciones públicas, en relación al fomento de la igualdad entre mujeres y hombres y el avance de la situación socioeconómica, política y cultural de la mujer" (Lombardo, 2004:30). Tienen que ver, por lo tanto, con las intervenciones de los poderes públicos encaminadas a eliminar las desigualdades entre mujeres y hombres y a mejorar la situación de las primeras.

En el marco de las políticas de igualdad de género se pueden distinguir tres grandes grupos o estrategias políticas de género que marcan la propia evolución y efectividad de las mismas. Estas son: las políticas de igualdad de trato, las acciones positivas y el mainstreaming.

1 En este sentido, el Instituto de la Mujer representó un modelo para la institucionalización autonómica de organismos de igualdad en el momento de desarrollo y profundización autonómica. Actualmente es un organismo autónomo dependiente del Ministerio de Igualdad, a través de la Secretaría General de Políticas de Igualdad. 


\section{CUADRO 1}

Tipología de políticas públicas de igualdad de género

\begin{tabular}{|l|l|l|l|}
\hline \multicolumn{1}{|c|}{ Estrategia } & \multicolumn{1}{|c|}{ Diagnosis } & \multicolumn{1}{c|}{ Solución } & \multicolumn{1}{c|}{ Responsabilidad } \\
\hline ¿quá está mal? & $\begin{array}{l}\text { ¿Qué hay que hacer? } \\
\text { Igualdad de trato } \\
\text { la ley }\end{array}$ & $\begin{array}{l}\text { Cambio de las leyes } \\
\text { para garantizar la } \\
\text { igualdad formal }\end{array}$ & Legisladores/as \\
\hline $\begin{array}{l}\text { Acciones específicas } \\
\text { para la igualdad }\end{array}$ & $\begin{array}{l}\text { Desigualdades de } \\
\text { partida. Problemas } \\
\text { específicos de las } \\
\text { mujeres y } \\
\text { desventajas }\end{array}$ & $\begin{array}{l}\text { Diseño y aplicación } \\
\text { de programas } \\
\text { específicos para las } \\
\text { mujeres }\end{array}$ & $\begin{array}{l}\text { Agencias } \\
\text { especializadas }\end{array}$ \\
\hline $\begin{array}{l}\text { Mainstreaming } \\
\text { de género }\end{array}$ & $\begin{array}{l}\text { Políticas habituales e } \\
\text { instituciones causan } \\
\text { o perpetúan la } \\
\text { desigualdad }\end{array}$ & $\begin{array}{l}\text { Reorganizar el } \\
\text { proceso político } \\
\text { para tener en cuenta } \\
\text { y tentar la } \\
\text { erradicación las } \\
\text { desigualdades. }\end{array}$ & $\begin{array}{l}\text { Gobierno y actores } \\
\text { implicados en los } \\
\text { procesos políticos }\end{array}$ \\
\hline
\end{tabular}

Fuente: Alonso (2007a) a partir de Verloo (2001).

Las políticas de igualdad de trato tienen que ver con aquellas medidas cuyo objetivo es alcanzar la igualdad formal de ambos sexos. En este sentido, buscan eliminar de la legislación existente posibles preceptos que discriminen explícitamente a alguno de los dos sexos. Son políticas deudoras de la concepción de raigambre liberal de igualdad de iure que ha sido objeto de importantes cuestionamientos debido a que pone entre paréntesis el reconocimiento de derechos que puedan ser específicos para alguno de los sexos (Mazey, 2001). No obstante, y a la luz de los resultados, el reconocimiento del principio de igualdad formal -doctrina liberal jurídica-no implicó la desaparición de todas las discriminaciones debido a que las condiciones sociales, económicas y políticas de partida de hombres y mujeres no eran iguales. En este sentido, muy pronto se reconoció que las políticas de igualdad de trato llevadas a cabo mayoritariamente durante los años setenta y principios de los ochenta no fueron eficaces ni suficientes para eliminar la desigualdad entre hombres y mujeres.

Las "acciones específicas" surgen como respuesta al déficit de igualdad de las primeras políticas de igualdad de trato. Las acciones específicas o acciones positivas suponen asumir la necesaria incorporación de un tratamiento diferenciado que posibilite la igualdad de oportunidades entre mujeres y hombres. La nueva denominación obedece a un cambio de enfoque hacia un concepto más amplio de igualdad de facto. Son estrategias que van más allá de la igualdad jurídica o formal hasta lo que requiere la igualdad real, esto es, la interposición de las medidas necesarias para que la igualdad sea efectiva, removiendo las barreras tanto culturales, económicas, como de cualquiera otro tipo que obstaculicen su consecución. Se pueden distinguir dentro de ellas las

148 denominadas políticas de igualdad de oportunidades y las políticas de acción positiva. 
Las primeras comprenden la eliminación de barreras legales, económicas, sociales, culturales y de poder para que mujeres y hombres tengan las mismas oportunidades $^{2}$. El segundo tipo de estrategia, las acciones positivas, o también denominadas de discriminación positiva tienen por objetivo corregir la desventaja inicial de las mujeres con el fin de conseguir la igualdad real de hombres y mujeres mediante el fomento de la presencia de estas últimas en los más diversos ámbitos. Así, se entiende como "acciones positivas"3 aquellas medidas extraordinarias que permiten a las mujeres conseguir la igualdad efectiva. En el marco de estas acciones se puede establecer una subdivisión entre las denominadas "acciones positivas" entendidas como la promoción de un trato preferente, para conseguir la igualdad a medio plazo y las denominadas medidas de "discriminación positiva" que implicarían el establecimiento de cuotas encaminadas a conseguir una igualdad inmediata (Barreme, 1997:89). Han sido estas últimas las medidas más controvertidas, ya que cuestionan la neutralidad de los diferentes procesos de selección. No obstante, estas acciones generalmente se han considerado como medidas temporales, es decir, entendidas como medios más que fines.

Por último, las políticas de mainstreaming constituyen la estrategia de más reciente aplicación. Esta estrategia fue asumida explícitamente por la Plataforma para la Acción de la Cuarta Conferencia Mundial sobre Mujeres de Naciones Unidas celebrada en Pekín en 1995. El Consejo de Europa la define así: “El mainstreaming de género es la organización (reorganización), el avance, el desarrollo y la evaluación de los procesos políticos, de modo que una perspectiva de igualdad de género, se incorpore en todas las políticas, a todos los niveles y en todas las etapas, por los actores normalmente involucrados en la adopción de medidas política" (Consejo de Europa, 1999).

El mainstreaming es la única estrategia que reconoce que la desigualdad y las relaciones de género se encuentran omnipresentes. Teniendo en cuenta que las actuaciones de las instituciones públicas no son neutrales respecto al género sino que forman parte activa del mantenimiento de la desigualdad estructural, esta nueva política tiene por objetivo introducir la perspectiva de género desde la propia definición del problema público que se intenta resolver, como a la hora de formular, ejecutar y evaluar las acciones públicas. Debe poder aplicarse en todas las políticas, a todos los niveles, en todas las etapas, y tener capacidad para reorganizar la práctica política. Se entiende como una nueva forma de hacer política de género que permite una aplicación flexible y adaptada a la realidad del ámbito donde se desarrolle.

Por otra parte, el mainstreaming como estrategia emplea diversas metodologías e instrumentos de intervención en materia de género, de modo que la revisión de las

2 El objetivo general de las mismas es el acceso de las mujeres al mundo público y su desarrollo se viene llevando a cabo por organismos especializados. Las más importantes están relacionadas con las políticas educativas y el acceso a formación para el mercado laboral.

3 El Consejo de Europa las define como aquellas medidas para "favorecer el acceso de miembros de ciertas categorías de personas, en este caso concreto, las mujeres, a los derechos que se le garantizó, en la misma medida que los miembros de otras categorías, en este caso, los hombres" (Consejo de Europa, 2004:54). Traducción propia. 
políticas públicas puede llevarse a cabo por procedimientos muy diversos, entre los que se encuentran los informes de impacto de género, los planes de igualdad y los presupuestos de género. En primer lugar, los “informes de impacto de género" son empleados en numerosos países y constituyen un tipo de evaluación ex-ante de las políticas públicas, por la cual antes que estas sean llevadas a la práctica, se analiza sus potenciales efectos sobre hombres y mujeres y su posible contribución a la permanencia de las desigualdades. En segundo lugar, los "planes de igualdad" constituyen un conjunto de medidas aprobadas por un gobierno para ser aplicadas por los departamentos que lo componen en un determinado período temporal (Bustelo, 1999: 381). Bajo esta herramienta y con el objetivo de incidir en la igualdad de género se programan una serie de actuaciones públicas que no son directamente aplicadas por el organismo de igualdad, sino que requiere la participación de otros actores. En tercer lugar, los presupuestos sensibles al género permiten hacer un análisis del presupuesto público para comprender el conjunto o partes del mismo, con el objetivo de conocer el alcance e impacto de las políticas públicas sobre las mujeres y niñas en comparación con los hombres y niños ${ }^{4}$. Son un mecanismo importante para asegurar una mayor consistencia entre los objetivos económicos y los compromisos sociales y políticos. Todas las políticas públicas que están o pueden estar reflejadas en el presupuesto pueden analizarse desde el punto de vista de género.

Por último y en otro orden de cosas, es importante subrayar que el mainstreaming de género no sustituye a las acciones específicas de igualdad de género correspondientes a la segunda generación de políticas de género. Éstas tienen por objetivo cubrir necesidades prácticas que afectan a la mayoría de las mujeres, destinatarias de acciones positivas. La implementación de acciones específicas, bien políticas de igualdad de oportunidades, bien acciones positivas permite dar a conocer la situación social que afecta a las mujeres, hacerla visible. Por lo tanto, estas últimas son condición necesaria pero no suficiente para la plena equiparación social, política y económica de mujeres y hombres. Son estrategias complementarias que forman parte del denominado sistema dual formado por las acciones específicas de igualdad y las políticas de mainstreaming.

\section{LA IGUALDAD DE GÉNERO EN GALICIA: ACCIONES, ESTRUCTURAS Y PRESENCIA}

El desarrollo de las políticas de género en la Comunidad Autónoma de Galicia, aunque un poco más tardío5, ha sido paralelo a los avances en diferentes institucio-

$4 \quad$ Un aspecto fundamental del análisis del presupuesto sensible al género es el reconocimiento del trabajo no remunerado de cuidado llevado a cabo mayoritariamente por mujeres mediante el uso del tiempo y de los recursos en los hogares. En este sentido, es importante reconocer la interdependencia de los sectores productivo y reproductivo de la economía y el carácter prioritario de los objetivos del bienestar y desarrollo humano que visibilicen las mujeres en la macroeconomía. Consúltese: (De la Fuente, M. y Ortiz, L., 2006).

5 Este desarrollo más tardío constituye el reflejo de la asunción competencial realizada en el Estatuto gallego. Con posterioridad a la creación del Emakunde vasco o el Instituto Andaluz de la Mujer en el 
nes europeas y españolas. A partir de los años 90 se han llevado a cabo diversas actuaciones públicas en materia de igualdad entre mujeres y hombres, actuaciones que han progresado siguiendo en gran medida a la lógica evolutiva de las distintas políticas de género descritas en los apartados anteriores pero también condicionadas por el color político del gobierno.

La ley 3/1991, de 14 de enero, de creación del Servicio Gallego de Promoción de la igualdad del hombre y de la mujer (SGI) fue debatida y aprobada en el Pleno del Parlamento de Galicia el 18 de diciembre de 1990. Su aprobación supuso el punto de partida del feminismo institucional en la comunidad gallega al crear el primer organismo específico cuya finalidad principal ha sido la de introducir las políticas de igualdad a nivel autonómico.

Entre las razones que llevaron al primer gobierno popular presidido por Fraga Iribarne a la creación del SGI, además de las señaladas en otras investigaciones como mimetismo institucional e influencia del contexto europeo (Alonso, 2007a; Bustelo, 2004; Mahou y Bouzas, 2007), cobra un peso significativo la postura que en la oposición había mantenido este partido en la anterior legislatura. Durante el gobierno tripartido de la segunda legislatura, las iniciativas parlamentarias de demanda de creación de un organismo de igualdad autonómico fueron apoyadas por el grupo parlamentario popular en la oposición. Una vez llegado al poder, la marcha atrás se hacía difícil.

Desde comienzos de los años 90 hasta el año 2005, el Partido Popular llevó a cabo políticas de igualdad de género del mismo modo que ha ocurrido en otras autonomías. En este sentido, a diferencia de otros contextos, estas políticas han sido paulatinamente aceptadas en España (Orbals, 2008; Threlfall, 1996), incluso en administraciones más conservadoras ${ }^{6}$. No obstante, esto no significa que exista una coincidencia en el resultado y contenido de las políticas de género 7 . De acuerdo con Candice Ortbals el color político del gobierno en Galicia ha marcado una diferencia significativa entre los casos gallego y andaluz, pues ha implicado un alejamiento de las demandas feministas y unas políticas públicas centradas en el rol de madre tradicional de la mujer y en el marco de la familia (Orbals, 2008).

año 88, la Xunta de Galicia inicia la aplicación de las competencias en materia de igualdad contenidas en el Estatuto de Autonomía (art. 4.2). Este marco estatutario le otorga competencias a la entidad autónoma gallega para actuar en materia de igualdad de género.

6 En general los partidos conservadores han incrementado sus actitudes favorables a las demandas feministas de igualdad de género como consecuencia de aspectos diversos como la necesidad de competir con la izquierda por el voto femenino, la creciente influencia de la legislación supranacional europea así como una mayor incorporación de las mujeres a la política (Ruiz Jiménez, 2002: 239).

7 Los contenidos de género requieren una matización a la hora de reconocer los intereses de mujeres: normalmente, los temas de género más próximos a posicionamientos de mujeres de derechas comprenden la defensa de la familia, la igualdad de oportunidades, el empleo y la lucha contra la violencia de género; mientras que los asuntos relativos a la legalización del aborto, los métodos anticonceptivos, la educación sexual o las cuotas son demandas más específicas de mujeres de un espectro ideológico de izquierdas (Lois y Diz, 2006). 
A partir del cambio en el gobierno en el año 2005 y hasta principios de 2009 destacan dos aspectos importantes para el análisis de la evolución de las políticas de igualdad. Por un lado, el gobierno es ocupado por dos partidos de izquierda -Partido Solicialista de Galicia (PSG) - Partido Socialista Obrero Español (PSOE) y Bloque Nacionalista Galego (BNG)- con una importante tradición de defensa de los intereses de las mujeres. Por otro lado, al tratarse de un gobierno bipartito, los dos partidos han intentado liderar la política de igualdad desde sus áreas de gobierno, lo que ha contribuido a una mayor presencia de la misma en la agenda política.

En estos momentos, a mediados del 2010, todavía resulta precipitado hacer una valoración profunda sobre las implicaciones que la vuelta del Partido Popular a la Xunta ha tenido en la evolución de las políticas de igualdad. Con todo, es posible subrayar algunos cambios significativos que dan como resultado un escenario con luces y sombras que hacen pensar que el factor político está directamente relacionado con el nuevo giro de las políticas gallegas de género.

\section{Acciones}

El impulso de las políticas públicas de igualdad de género, esto es, de las medidas destinadas a eliminar las discriminaciones contra las mujeres, se ha concretado fundamentalmente a través de los Planes de Igualdad de oportunidades. Los planes de igualdad, como se indicó más arriba, constituyen un conjunto de medidas aprobadas por un gobierno para ser aplicadas por los departamentos que lo componen en un determinado intervalo temporal (Arranz, 2000: 39). Son el principal instrumento de actuación de los organismos que trabajan para la eliminación de las relaciones desiguales de género. Desde la creación del SGI, se han puesto en funcionamiento cinco Planes de Igualdad:

I Plan para la igualdad de oportunidades de las mujeres gallegas, 1992-1994

II Plan para la igualdad de oportunidades de las mujeres gallegas, 1995-1997

III Plan para la igualdad de oportunidades de las mujeres gallegas, 1998-2001

IV Plan para la igualdad de oportunidades de las mujeres gallegas, 2002-2005

V Plan del Gobierno gallego para la igualdad entre mujeres y hombres, 2007-2010

El título del V Plan de igualdad, vigente en la actualidad, da cuenta de un salto cualitativo respecto a las políticas de género por dos motivos. En primer lugar, y por primera vez, nos encontramos delante de un Plan del Gobierno gallego. Los planes anteriores fueron aprobados por el Pleno del Consejo del SGI, lo que significaba un compromiso menor por parte del gobierno del momento y una mayor dificultad para lograr que otros departamentos se sintieran forzados a la hora de cumplir las medi-

152 das recogidas en los planes. En segundo lugar, la alusión explícita a mujeres y hom- 
bres como destinatarios del V Plan, frente a la referencia sólo a las mujeres en los anteriores, evidencia el cambio de perspectiva a la hora de entender las políticas de igualdad como algo que beneficia a la sociedad en su conjunto y que necesariamente tiene que implicar también a los hombres.

En esta misma línea, si se observan con detalle los contenidos de los planes, es posible identificar otro de los aspectos significativos de la evolución de las políticas de igualdad en Galicia, a saber, la adopción de la estrategia del mainstreaming.

Como se indica en el cuadro 2 referido a las áreas de actuación de los planes gallegos de igualdad, el mainstreaming se incorpora por primera vez en el III Plan de Igualdad. Con todo, mientras que la mayor parte de los planes combinan políticas específicas con el nuevo mainstreaming, el V Plan avanza cualitativamente en esta estrategia diseñando líneas de actuación genéricas que persiguen el cambio de las condiciones estructurales que condicionan la persistencia de la desigualdad en las sociedades actuales ${ }^{8}$.

\section{CUADRO 2}

Tipología de áreas de actuación en los planes gallegos de igualdad

\begin{tabular}{|l|l|l|l|l|l|}
\hline \multicolumn{1}{|c|}{ Áreas de actuación } & I & II & III & IV & V \\
\hline Participación sociopolítica & & & & & \\
\hline Sensibilización & & & & & \\
\hline Medios de Comunicación & & & & & \\
\hline Educación & & & & & \\
\hline Formación y empleo & & & & & \\
\hline Conciliación & & & & & \\
\hline Cultura & & & & & \\
\hline Deporte & & & & & \\
\hline Desarrollo sostenible & & & & & \\
\hline Salud & & & & & \\
\hline Servicios sociales & & & & & \\
\hline Violencia de género & & & & & \\
\hline Mainstreaming & & & & & \\
\hline
\end{tabular}

Fuente: Elaboración propia a partir de Alonso (2007a).

Junto con estos Planes de igualdad de contenido general nos encontramos con tres planes específicos que podemos enmarcar en la estrategia del mainstreaming: El

8 Las líneas estratégicas de este $V$ plan son cuatro: participación y empoderamiento; acceso a información y a los recursos; formación en igualdad entre mujeres y hombres; y tratamiento integral de la violencia de género. 
Plan de Fomento de la Corresponsabilidad y el Plan para la Salud integral de las Mujeres, aprobados los dos en el 2007 y el Programa Gallego de Mujer y Ciencia, aprobado en el año 2008.

Asimismo, otras acciones relevantes en Galicia en materia de igualdad han sido la aprobación de distintas leyes autonómicas. Nos referimos a la Ley Gallega 7/2004, de 16 de julio, para la igualdad de mujeres y hombres; a la Ley 2/2007, de 28 de marzo, del trabajo en igualdad de las mujeres de Galicia y a la Ley 11/2007, de 27 de julio, gallega para la prevención y el tratamiento integral de la violencia de género. La aprobación de medidas legislativas supone un tipo de intervención que apuesta por herramientas de actuación más incisivas y más comprensivas de lo que resultan los planes (Aránzazu, 2003: 67; Bustelo y Ortbals, 2007: 211). Y es que una de las limitaciones de los planes de igualdad radica en el hecho de que las políticas tienden a permanecer exclusivamente en el ámbito del ejecutivo. Por el contrario, las leyes permiten que la acción en género se amplíe al ámbito legislativo y judicial. Además, en el caso gallego, esta estrategia va a suponer la adopción del mainstreaming como principio de actuación de los poderes públicos gallegos.

La Ley Gallega 7/2004, de 16 de julio, para la igualdad de mujeres y hombres en la Comunidad Autónoma de Galicia establece el principio de mainstreaming en la elaboración, en la ejecución y el seguimiento de todas las políticas y de todas las actuaciones de la Xunta de Galicia. Junto con esta disposición general, la ley incorpora a la actuación de la administración autonómica alguno de los instrumentos del mainstreaming:

- En el capítulo II, dedicado al principio de mainstreaming, introduce dos artículos, el séptimo y el octavo, referidos a los informes de impacto de Género en la elaboración de las leyes y de las legislaciones elaboradas por la Xunta de Galicia. Los informes de impacto se establecen de manera obligatoria, aunque no vinculante, para proyectos y proposiciones de ley.

- En el capítulo III se regula la erradicación del uso sexista del lenguaje. En él se define el lenguaje no sexista y se obliga a la Xunta a la supresión del mismo en el campo institucional; al mismo tiempo se establece que la administración gallega ha de procurar su eliminación en el campo social.

- Por último, la disposición adicional primera fija, con la finalidad de facilitar la aplicación del principio de mainstreaming, que las estadísticas e investigaciones con eventual repercusión en cuestiones de género realizadas por la comunidad autónoma se encuentren desagregadas por sexos.

Luego, pese a que los instrumentos que regula, así como su descripción y definición no consiguen la profundización necesaria para la optimización de la implementación del mainstreaming, la Ley 7/2004 supone un paso importante en la incorporación 154 de esta estrategia en la actuación de la Xunta de Galicia. 
En el año 2007 la actuación legislativa en materia de igualdad da un paso significativo en la incorporación del mainstreaming a la acción de la administración autonómica. Este avance obedece a la incorporación del objetivo de la igualdad como dimensión central en la actuación de políticas sectoriales: violencia y trabajo. Así, la Ley $2 / 2007$, de 28 de marzo, del trabajo en igualdad de las mujeres de Galicia, en su artículo primero, regula que su objeto es la integración de la dimensión de la igualdad de oportunidades entre mujeres y hombres en las competencias autonómicas sobre empleo y relaciones laborales. De la misma manera, la Ley 11/2007, de 27 de julio, gallega para la prevención y el tratamiento integral de la violencia de género establece como objetivo la adopción de medidas integrales dirigidas a la prevención y atención del tratamiento de la violencia ejercida contra las mujeres, así como la asistencia a las víctimas. Esta ley pretende dotar a los poderes públicos y a la sociedad gallega de un instrumento útil para prever, tratar y erradicar la violencia de género y, en fin, conseguir una verdadera realización del principio de igualdad.

Como se ha señalado anteriormente, uno de los aspectos positivos de la introducción del mainstreaming en estas leyes es el carácter obligatorio otorgado a alguna de sus herramientas -estadísticas, informes de impacto o lenguaje no sexista-. Por ello, antes de dar por finalizado este apartado resulta pertinente el examen de alguna de ellas. En concreto, la referida a los informes de impacto de género que, como ya se ha indicado, se introducen en la ley del 2004. Los informes han sido la principal herramienta del gobierno gallego para incorporar la perspectiva de género en su legislación. Un reciente estudio (Alonso, Diz y Lois, 2009) evalúa cuál ha sido el verdadero impacto de estos informes y da cuenta de su utilización no sólo en las leyes, tal y como obliga el artículo séptimo en el que se regula, sino en otro tipo de normativa. Además, en cuanto a su influencia, se constatan dos tendencias. En primer lugar, no todas las áreas de gobierno han sido igualmente receptivas a la inclusión de este tipo de recomendaciones. $Y$, en segundo lugar, cuanto más transformadoras han sido las sugerencias presentes en los informes menos posibilidades ha habido de que éstas fueran incluidas finalmente en la normativa. Es más, el estudio subraya la importancia de la existencia de una normativa en igualdad, ya que son las medidas de igualdad que están legisladas las que finalmente se incorporan en otra legislación. Con todo, se puede concluir que estamos ante una herramienta bastante consolidada en el ámbito de actuación de la Xunta de Galicia.

En último lugar, y como resultado del cambio de gobierno, es preciso destacar nuevas acciones recogidas en el Anteproyecto de Ley de apoyo a la Familia y a la Convivencia9. En él se dilucidan nuevas orientaciones entre las que destacan las denominadas acciones de "apoyo a la vida" que hacen pensar en un claro cambio de rumbo respecto a las políticas de género de la legislatura anterior. En realidad, es un proyec-

9 El 19 de mayo de 2010 la Conselleira de Traballo e Benestar presentó a la prensa el borrador de la futura Ley de apoyo a la Familia y a la Convivencia. 
to que guarda una relación muy estrecha con las recientes leyes autonómicas de Castilla y León y Valencia gobernadas por el PP10.

Al parecer, esta nueva ley tendrá presente el "apoyo a la vida" con medidas hacia las mujeres gestantes con el fin de fomentar que el embarazo llegue a término. En este sentido, las acciones girarán en torno al "fomento del acogimiento familiar y de la adopción”. Estas propuestas de actuación se encuentran en clara sintonía con algunas claves de las políticas en contra del aborto manifiestamente opuestas a las demandas feministas.

Por otro lado, como elemento novedoso, esta futura ley considerará a las mujeres embarazadas como unidad familiar de pleno derecho y creará por primera vez en la Comunidad un registro de familias monoparentales. Con esta iniciativa legislativa se tiene por objetivo sentar las bases del Plan Gallego de Apoyo a la Familia y a la Natalidad que permitirá la creación de un Consello Galego das Familias.

\section{Estructuras}

El mainstreaming supone la creación y transformación de la organización de las estructuras administrativas con el objetivo de garantizar la introducción de la perspectiva de género en todo el proceso de elaboración de una política pública concreta. ¿De qué estructuras se trata? Por un lado, la necesaria estrategia dual concede un papel decisivo a los organismos de igualdad tradicionales o instituciones de igualdad específicas. Por otro lado, las nuevas estructuras deben dar respuesta a algunos de los requisitos del mainstreaming, de manera que se creen: 1. Órganos específicos tendentes a favorecer una mayor coordinación y colaboración institucional, 2. Órganos de consulta igualitaria dirigida a la integración de la sociedad civil en el proceso de cambio y la puesta en práctica de políticas participativas y 3. Órganos que permitan la extensión de los objetivos de la igualdad de género hacia nuevas áreas competenciales.

En cuanto a los organismos de igualdad tradicionales, algunas autoras han señalado (Bustelo, 2004; Bustelo y Ortbals, 2007) dos elementos estructurales que se deben utilizar a la hora de explicar su fuerza institucional: el tipo de organismo -referido a si es un organismo autónomo, con presupuesto propio y creado por ley, o si se trata, al contrario, de una dirección general, dependiente de un departamento del gobierno y de sus decisiones- y el departamento o área gubernamental en el que está situado. De acuerdo con estas autoras, el área de gobierno preferible es la de presidencia ya que, por un lado, sus competencias se extienden a otras áreas de gobierno

10 Son la Ley 6/2009, de 30 de junio, de la Generalitat, de Protección a la Maternidad y la Ley $14 / 2008$, de 18 de diciembre, por la que se crea y regula una red de apoyo a la mujer embarazada. Al parecer, el origen de estas leyes se encuentra en la iniciativa legislativa popular de la Fundación Red Madre. Es una red de asociaciones de asesoramiento a embarazadas impulsada en 2007 por el Foro Español de la 156 mentos autonómicos elaboren normas de apoyo a la maternidad. 
y, por otro, otorga a las femócratas o feministas de Estado una mejor situación para persuadir a los colegas de otros departamentos.

El diseño orgánico del SGI, así como otros organismos de igualdad autonómicos, siguen en su creación el modelo de la administración central: un organismo autónomo vinculado a un departamento percibido como de importancia secundaria, esto es, Trabajo y Servicios Sociales.

Con todo, la consejería de adscripción del SGI fue cambiando a lo largo de los años de manera que se experimenta una evolución positiva desde el punto de vista de la autoridad y de la capacidad del feminismo institucional en el nivel autonómico. La evolución cronológica de los órganos de adscripción ha sido la siguiente:

1. Consejería de Trabajo y Servicios Sociales.

2. Consejería de Familia Mujer y Juventud.

3. Consejería de promoción del Empleo, Mujer y Juventud.

4. Consejería de Familia, Juventud, Deporte y Voluntariado.

5. Vicepresidencia de la Igualdad y el Bienestar (Secretaría General de Igualdad).

6. Presidencia (Secretaría General de lgualdad).

Esta evolución de la vinculación de las responsabilidades en igualdad de género y del SGI a los diferentes departamentos determina un distinto peso político y capacidad de influencia en el establecimiento de la agenda. Se puede establecer una división clara entre dos grandes períodos en lo referido al nivel organizativo. Un primero, antes del 2005, donde las políticas de género se sitúan en consejerías de bajo nivel de influencia y que actúan en ámbitos de poca incidencia para las restantes unidades de gobierno.

La estructura posterior al 2005 representa, por el contrario, un salto cualitativo y abre otra etapa. El Servicio Galego de Igualdade se adscribe en este periodo al entorno de Presidencia. Este nivel, como se acaba de indicar, determina la presencia en un plano ventajoso pues constituye un nivel privilegiado para la introducción de la estrategia de la mainstreaming, ya que favorece capacidad de influencia y de coordinación en otros organismos para que incluyan la igualdad entre sus objetivos.

Desde este último punto de vista, esto es, el de la introducción del mainstreaming, también es preciso explicar la novedad que ha representado, en cuanto a diseño estructural de las políticas de igualdad, la adscripción de esta área a una secretaría general incardinada en el gobierno. Sin duda, la capacidad ejecutiva de una secretaría general, frente a un organismo autónomo, significa situar en un primer plano la igualdad en la agenda política. Por otro lado, el avance que las políticas de igualdad en 
Galicia han experimentado desde el 2005 podrían estar indicando la utilidad de este tipo de estrategia estructural. Con todo, se necesitan todavía estudios cualitativos que indaguen en esta relación, así como en las consecuencias que la aparición de estas estructuras puede tener sobre los organismos de igualdad tradicionales ${ }^{11}$.

La normativa aprobada por el gobierno gallego a partir del 2007 da respuesta a través de la creación de distintas estructuras a alguno de los desafíos del mainstreaming. En este sentido, en Galicia las femócratas han utilizado la ley como una oportunidad para impulsar esta estrategia en una de sus principales dimensiones, a saber, el cambio de la estructura administrativa para la igualdad. Sintetizando y de manera esquemática se puede describir este nuevo entramado institucional como sigue:

En la nueva normativa, a las unidades especializadas de igualdad -Servicio Gallego de promoción de la Igualdad del hombre y de la mujer y, sobre todo, Secretaría General de la lgualdad- se les sigue reconociendo sus funciones y se profundiza en alguna de ellas.

Con respeto a otros cambios estructurales, se crea un organismo de coordinación entre consejerías: Comisión Interdepartamental de Igualdad. La creación de esta comisión que ya funcionó para la elaboración del V Plan del Gobierno gallego de igualdad entre mujeres y hombres de Galicia, se regula en la Ley 11/2007, de 27 de julio, gallega para la prevención y el tratamiento integral de la violencia de género como un órgano colegiado e institucional de la Xunta.

La participación en el proceso de elaboración de las políticas públicas de igualdad se garantiza con la creación de dos organismos: el Consejo gallego de las mujeres y Consejo gallego de participación de las mujeres en el ámbito del empleo y de las relaciones laborales. La Ley 11/2007, de 27 de julio, gallega para la prevención y el tratamiento integral de la violencia de género crea el Consejo Gallego de las Mujeres como órgano colegiado e institucional de Galicia, de carácter consultivo, de participación y asesoramiento en materia de políticas de igualdad. Además, la Ley 2/2007, de 28 de marzo, del trabajo en igualdad de las mujeres de Galicia también regula el Consejo Gallego de participación de las mujeres en el ámbito de empleo y de las relaciones laborales, como órgano de participación mediante un canal de libre adhesión de las asociaciones de mujeres, donde se integren las secretarías de mujeres de los sindicatos y de la confederación de empresarios y empresarias de Galicia y con representación del departamento de la administración autonómica en materia de igualdad.

11 En el caso gallego, durante el gobierno bipartito el SGI fue adscrito a la Secretaría General de Igualdad y ambos contaban con una dirección nombrada, lo que suponía, a los ojos de la ciudadanía, la percepción de dos liderazgos en política de igualdad. El gobierno actual del Partido Popular parecía haber optado, en un primer momento, por mantener un único liderazgo. En este sentido, el caso del ejecutivo central, con la creación del Ministerio de Igualdad, resulta un cambio estructural similar donde se impone también la indagación sobre el papel del Instituto de la Mujer. 
En otro orden de cosas, con respeto a las estructuras especializadas de área, en los últimos años se crean dos: el Servicio de igualdad laboral y la Unidad Mujer y Ciencia.

El Servicio de igualdad laboral se regula en la Ley 2/2007, de 28 de marzo, del trabajo en igualdad de las mujeres de Galicia. Se trata de la Unidad Administrativa de Igualdad del departamento de la Administración autonómica competente en materia de trabajo, que funcionará coordinada y vinculada funcionalmente con el departamento de la administración autonómica competente en materia de igualdad. Las funciones que se le atribuyen son de asesoramiento en materias de género, impulso de la participación equilibrada de mujeres y hombres en los puestos de trabajo del citado departamento, diseño de formación en materia de igualdad, colaboración con el departamento competente en materia de igualdad en la elaboración de los informes de impacto de género de las normas del departamento de la administración autonómica competentes en materia de trabajo, recepción de las estadísticas que este elabora, promoción del principio de igualdad, asesoramiento a las empresas, revisión de convenios colectivos, elaboración de dictámenes y vigilancia sobre el cumplimiento de esta ley.

Asimismo, se debe mencionar la creación, a través del Decreto 33/2007 del 1 de marzo, de la Unidad de Mujer y Ciencia. Se trata de un organismo especializado en potenciar la presencia de las mujeres en los ámbitos científico y tecnológico y que cuenta con la participación de hasta tres departamentos gubernamentales: la Secretaría General de Igualdad, la Consellería de Educación y la Consellería de Innovación e Industria. La colaboración de estas dos últimas representa un claro ánimo para la aplicación del mainstreaming, ya que entran a formar parte del feminismo institucional, mostrando un claro compromiso con la eliminación de las desigualdades y aportando su experticia (Alonso, 2008a; 2008b).

Por último, y con el cambio de gobierno en la primavera de 2009, resulta pertinente destacar el reciente Anteproyecto de Ley por el que se suprime el Servicio Galego da Igualdade y se modifican determinados artículos ${ }^{12}$ de la Ley 2/2007, de 28 de marzo, del trabajo en igualdad de las mujeres. Tras diecinueve años de funcionamiento de este organismo que significó el punto de arranque del feminismo institucional en Galicia será suprimido aludiendo a criterios de racionalización de las estructuras. En este anteproyecto se argumenta que con la concentración de las funciones en la ya existente Secretaría General de Igualdad se gana en eficacia a la hora de aplicar el mainstreaming, sin embargo, no está claro que un organismo de rango menor posea un gran poder de persuasión sobre las consejerías. Los organismos autónomos, como el SGI, se valoran como los más adecuados y extendidos en el conjunto de las autonomías ${ }^{13}$ ya que garantizan la continuidad y la estabilidad de las políticas de género al tiempo que impiden que éstas sufran grandes retrocesos con los cambios de gobierno.

12 Se modifica el artículo 7 rebajando a la "Unidad Administrativa de Igualdad", adscrita hasta el momento a la Secretaría General de la Consellería de Traballo, a la condición de servicio.

13 La organización exclusivamente política -direcciones generales en su mayoría- está presente tan solo en los casos de Cantabria, Valencia, Madrid y Castilla-León. 


\section{Presencia}

Hasta ahora se han abordado dos de las tres estrategias utilizadas para incrementar la posición social de las mujeres (Squires, 2007): la creación de organismos de igualdad -con el objetivo de lograr una representación substantiva de los intereses de las mujeres- y la estrategia del mainstreaming. Queda referirse a la utilización de las cuotas para garantizar la presencia de las mujeres en los puestos representativos.

A diferencia de otras leyes de igualdad autonómicas que introducen las cuotas como medida para garantizar la paridad en la selección de personas para puestos representativos de ámbito autonómico, la Ley gallega de igualdad del 2004 establecía el fomento de la participación equilibrada de las mujeres, pero no garantizaba la misma a través del establecimiento de cuotas. Es la ley orgánica, 3/2007, de 22 de marzo, para la igualdad efectiva entre mujeres y hombres la que obliga a la aplicación del principio de presencia equilibrada en las elecciones a los parlamentos autonómicos. Además, la Ley 2/2007, de 28 de marzo, del trabajo en igualdad de las mujeres de Galicia utiliza las disposiciones adicionales para la reforma de la Ley $1 / 1983$, de 22 de febrero, de normas reguladoras de la Xunta y su presidencia y establece, mientras que el Parlamento de Galicia no legisle otro porcentaje, el mínimo del $40 \%$ de presencia femenina en el ejecutivo gallego.

Como se puede observar en las tablas siguientes, los resultados de esta normativa han sido positivos aunque parciales. La presencia de parlamentarias en la cámara autonómica representa por primera vez en su historia el 40\%. El análisis de las listas y los puestos de las candidatas en las mismas, sin embargo, pone de manifiesto otra interpretación que ya ha sido señalada para otros contextos (Verge, 2008; Diz y Lois, 2009). El principio de presencia equilibrada que establece un mínimo del $40 \%$ y un máximo del 60\% para cada uno de los sexos, lejos de aplicarse como tal principio se utiliza sistemáticamente como una cuota del 40\% para las mujeres.

Por otro lado, en cuanto a la presencia de las mujeres en el ejecutivo, el porcentaje mínimo del $40 \%$ se cumple en el caso de conselleiras y conselleiros, pero no en los puestos de designación política que están lejos de alcanzar los porcentajes que establece la ley.

TABLA 1

Presencia de mujeres en el Parlamento de Galicia al inicio de cada legislatura, en porcentajes

\begin{tabular}{|l|c|c|c|c|c|c|c|c|}
\hline & $\begin{array}{c}\text { I LEG } \\
(\mathbf{8 1 - 8 5})\end{array}$ & $\begin{array}{c}\text { II LEG } \\
(\mathbf{8 5 - 8 9 )}\end{array}$ & $\begin{array}{c}\text { III LEG } \\
(\mathbf{8 9 - 9 3 )}\end{array}$ & $\begin{array}{c}\text { IV LEG } \\
(\mathbf{9 3 - 9 7})\end{array}$ & $\begin{array}{c}\text { V LEG } \\
(\mathbf{9 7 - 0 1 )}\end{array}$ & $\begin{array}{c}\text { VI LEG } \\
(\mathbf{0 1 - 0 5})\end{array}$ & $\begin{array}{c}\text { VII LEG } \\
(\mathbf{0 5 - 0 9 )}\end{array}$ & $\begin{array}{c}\text { VIII LEG } \\
(\mathbf{0 9}-)\end{array}$ \\
\hline BNG & - & - & $20 \%$ & $15,3 \%$ & $22,2 \%$ & $17,6 \%$ & $38,4 \%$ & $50 \%$ \\
\hline PP & $3,8 \%$ & $2,9 \%$ & $10,5 \%$ & $14 \%$ & $14,3 \%$ & $36,6 \%$ & $24,3 \%$ & $34,2 \%$ \\
\hline PSdeG & - & - & $17,8 \%$ & $10,5 \%$ & $23,1 \%$ & $35,3 \%$ & $40 \%$ & $44 \%$ \\
\hline Total & $4,2 \% *$ & $1,4 \%$ & $12 \%$ & $13,3 \%$ & $17,3 \%$ & $30,6 \%$ & $33,3 \%$ & $40 \%$ \\
\hline
\end{tabular}

Fuente: Elaboración propia a partir de datos del Diario Oficial de Galicia.

* En la I legislatura había otras 2 diputadas pertenecientes a UCD. 
TABLA 2

Presencia de mujeres en las listas en las elecciones al Parlamento de Galicia del 2009

\begin{tabular}{|l|c|c|c|c|c|}
\hline & $\begin{array}{c}\text { Presencia } \\
\text { listas }\end{array}$ & $\begin{array}{c}5 \text { primeros } \\
\text { puestos } \\
\text { (Composición } \\
3 \text { mujeres / } 2 \\
\text { hombres) }\end{array}$ & $\begin{array}{c}\text { Cabezas de } \\
\text { lista }\end{array}$ & $\begin{array}{c}\text { Puestos de } \\
\text { salida }\end{array}$ & $\begin{array}{c}\% \text { en el } \\
\text { parlamento }\end{array}$ \\
\hline Galicia & $48,8 \%$ & $25 \%$ & $25 \%$ & $40 \%$ & $40 \%$ \\
\hline
\end{tabular}

Fuente: Diz y Lois, 2009.

TABLA 3

Presencia de mujeres en el ejecutivo autonómico actual, en porcentajes

\begin{tabular}{|l|c|c|c|c|}
\hline & Presidencia & Consejerías & $\begin{array}{c}\text { Secretarías } \\
\text { generales }\end{array}$ & $\begin{array}{c}\text { Direcciones } \\
\text { generales }\end{array}$ \\
\hline$\%$ mujeres & - & 40 & $35 \%$ & $23 \%$ \\
\hline
\end{tabular}

Fuente: Novo, 2009.

\section{CONCLUSIONES}

En resumen, se puede concluir que, en términos generales, la Comunidad Autónoma gallega ha alcanzado en los últimos años un notable estado de madurez e institucionalización de las políticas de igualdad de género pues han sido ampliamente desarrolladas y equiparadas a las que podemos encontrar en las autonomías que más han avanzado en este campo e incluso respecto a las llevadas a cabo en el nivel estatal.

En los años 90 se ha producido un progreso continuo motivado en gran medida por la lógica evolutiva de las distintas estrategias de género aunque también el color político ha sido un factor significativo a la hora de entender el alcance y resultado de las políticas de género. A partir de la creación del SGI las políticas gallegas experimentaron una evolución constante. En primer término, se desarrollaron cómo instrumentos específicos de actuación los planes plurianuales de igualdad y normativa específica. La aprobación de los diferentes planes determinó una continua revisión y ampliación de la agenda política. Paralelamente, se han producido modificaciones en la estructura organizativa con la que se aborda la igualdad.

Ahora bien, el período de legislatura iniciado en 2005 con el gobierno bipartito supuso un salto cualitativo tanto respecto al contenido de las políticas como a las estructuras. Los nuevos planes e iniciativas legislativas se llevaron a cabo con una vocación de implementación completa del mainstreaming -en todas las áreas y en todas las políticas- creándose al mismo tiempo órganos y unidades ex-novo como la Secretaría General de Igualdad, la Comisión Interdepartamental de la Igualdad o la Unidad Mujer y Ciencia, entre otros. Pese a que estos cambios importantes pueden 
comprenderse también como fruto del proceso de consolidación de la estrategia estructural del mainstreaming iniciada a mediados de los años noventa, la voluntad política ha sido manifiesta.

A partir del 2009, con el regreso del PP al gobierno, y sin que haya sido posible llevar a cabo un análisis extenso debido al corto período de tiempo contemplado, se ha evidenciado un cambio de rumbo. Las transformaciones sustantivas en el contenido y alcance las políticas de género como la decisión de suprimir el SGI, organismo autónomo referente del feminismo institucional en Galicia, así como la modificación de la Ley 2/2007 del 28 de marzo del trabajo en igualdad de las mujeres, o el nuevo proyecto de ley sobre la familia y la convivencia, dibujan un horizonte de incertidumbre que podría estar poniendo en peligro algunos de los recursos institucionales gallegos necesarios para una agenda política de igualdad de género con garantía de futuro. En definitiva, habrá que esperar para saber si tras el camino abierto en los últimos años, se sigue haciendo camino al andar.

\section{REFERENCIAS BIBLIOGRÁFICAS Y DOCUMENTALES}

Alonso, A. (2007a), El mainstreaming y la europeización de las políticas de género en Galicia. Trabajo de investigación tutorado presentado en el programa de Doutoramento Procesos Políticos Contemporáneos. Curso 2006-2007. Santiago de Compostela: Facultad de Ciencias Políticas de la Universidad de Santiago de Compostela.

- (2007b), “El mainstreaming y la europeización de las políticas de género en Galicia”. Ponencia presentada en el VIII Congreso de la Asociación Española de Ciencia Política. En línea: <http://www.aecpa.eres/congreso_08/archivos/arena6/GT-19/ ALONSO-ALBA.pdf > (consulta: 21 diciembre 2009).

- (2008a), "Género y Ciencia. Una visión desde las políticas públicas gallegas", Andaina Revista del movimiento feminista gallego, 49: 47-50.

- (2008b), "La perspectiva de género en las políticas científicas: estudio de cuatro Comunidades Autónomas". Ponencia presentada en International Congress New and Old Questions of Women and Gender Research, CIFEX, 14 de mayo, Santiago de Compostela.

Alonso, A., Diz, I. y Lois, M. (2009), Os informes galegos de impacto de xénero: unha análise da súa incidencia na elaboración das normas, Santiago de Compostela: Servicio Galego de Igualdade, Informe-trabajo en línea: <http://sgi.xunta.es/document/infimpacxen.pdf> (consulta: 15 febrero 2010).

Aránzazu, M. (2003), “Las nuevas leyes autonómicas de igualdad de mujeres y hombres”, Aequalitas, 13: 63-74.

Arranz, F. (2000), “Algunas notas tecnológicas sobre los planes de Igualdad de Oportunidades en el marco de las políticas públicas dirigidas a mujeres: el caso español”, en F. Arranz, ed., Las políticas públicas a favor de las mujeres. Madrid: Instituto Estudios Feministas Universidad Complutense de Madrid.

162 Astelarra, J. (2005), Veinte años de políticas de igualdad. Madrid: Cátedra. 
Barréme, M. A. (1997), Discriminación, derecho antidiscriminatorio y acción positiva en favor de la mujeres. Madrid: Civitas.

Bustelo, M. (1999), "Políticas públicas de igualdad de género en España: evolución y evaluación”, en M. Ortega, C. Sánchez y C. Valiente, eds., Género y Ciudadanía. Revisiones desde el ámbito privado. Madrid: Ediciones de la Universidad Autónoma de Madrid.

- (2004), La evaluación de las políticas de igualdad de género. Madrid: Catarata.

Bustelo, M. y Ortbals, C. (2007), "The evolution of Spanish State Feminism: a Fragmented Landscape”, en J. Outshoorn y J. Kantola, eds., Changing State Feminism. Londres: Palgrave Macmillan.

Castro, C. (2003), Introducción al enfoque integrado del mainstreaming de género. Sevilla: Instituto Andaluz de la Mujer.

Comisión Europea (1999), Guía para la evaluación del impacto de género. Bruselas: European Commission, Employment, Social Affairs and Equal Opportunities. En línea: <http://ec.europa.yo/employment_social/gender_equality/docs/gender/ gender_eres.pdf.> (consulta: 20 febrero 2010).

Consejo de Europa (1999), Mainstreaming de género. Marco conceptual, metodología y presentación de buenas "prácticas". Informe final de las actividades del Grupo de especialistas en mainstreaming (EG-S-MS) (versión castellana e inglés). Madrid: Instituto de la Mujer, serie documentos, n. 28.

De La Fuente, M. y Ortiz, L., coords., (2006), Presupuestos locales con perspectiva de género. Barcelona: ICPS.

Diz, I. y Lois, M. (2009), “Implementando el principio de representación equilibrada: un análisis de los procesos de reclutamiento en los partidos políticos", ponencia presentada en el IX Congreso de la Asociación Española de Ciencia Política (AECPA), celebrado en Málaga en septiembre del 2009.

Fraser, N. (1997), Justice Interruptus, Critical Reflections on the "Postsocialist" Condition. Londres: Routledge.

Lodeiro, C. (2007), Políticas públicas de igualdad de género en el ámbito autonómico: el Servicio Gallego de la lgualdad del Hombre y de la Mujer. Trabajo de investigación tutorado presentado en el programa de Doctorado Procesos Políticos Contemporáneos. Curso 2006-2007. Santiago de Compostela: Facultad de Ciencias Políticas de la Universidad de Santiago de Compostela.

Lois, M. y Diz, I (2006), “¿Qué sabemos sobre la presencia política de las mujeres y la toma de decisiones? Claves para un marco de análisis", Política. Revista de Ciencia Política, 46: 37-60.

Lombardo, E. (2003) "El mainstreaming. La aplicación de la transversalidad en la Unión Europea", Aequalitas, 13: 6-11.

- (2004), La europeización de la política española de igualdad de género. Valencia: Tirant lo Blanch.

Lovenduski, J. (1997), “Feminismo institucional: género y estado”, en E. Uriarte y A. Elizondo, eds., Mujeres en Política. Barcelona: Ariel.

Mahou, X. y Bouzas, R. (2007), “Gobierno electrónico y organismos de igualdad: examen de los canales de acceso”, en I. Diz y M. Lois, eds., Mujeres, instituciones y política. Barcelona: Bellaterra. 
Mazey, S. (2001), Gender Mainstreaming in the EU. Principles and Practice. Londres: Kogan.

Novo, A. (2009), “Implementando la paridad en España: un análisis de los gabinetes”. Ponencia presentada en el XIV Congreso Internacional del CLAD, celebrado en Brasil en octubre del 2009.

Ortbals, C. (2008), "Subnational Politics in Spain: New Avenues for Feminist Policymaking and Activism", Politics and Gender, 4: 93-116.

Roggeband, C. y Verloo, M. (2006), "Evaluating gender impact assessment in the Netherlands (1994-2004): la political process approach", Policy and Politics, 34(4): 615-632.

Ruiz Jiménez, A. (2002), Mecanismos de cambio ideológico e introducción de políticas de género en partidos conservadores: el caso de AP-PP en España en perspectiva comparada. Madrid: Centro de Estudios Avanzados en Ciencias Sociales.

Squires, J. (2007), The New Politics of Gender Equality. Londres: Palgrave Macmillan

Stetson, D. y Mazur, A. (1995), Comparative State Feminism. Thousand Oaks, CA: Sage.

Threlfall, M. (1996), "Feminist Politics and Social Change in Spain", en M. Threlfall, Mapping the Women's Movement, Feminist Politics and Social Transformation in the North. Londres: Verso.

Verge, T. (2008), "Cuotas voluntarias y legales en España. La paridad a examen", Revista Española de Investigaciones Sociológicas, 123: 123-150.

Verloo, M. (2001), "Another velvet revolution? Gender Mainstreaming and the politics of Implementation”, IWM Working papers, n. ${ }^{\circ}$. Viena: IWM.

Valiente, C. (1996), "El feminismo institucional en España: el Instituto de la Mujer, 1983-1994”, Revista Internacional de Sociología, 13: 163-204.

Recibido: 29 de enero de 2010

Aceptado: 2 de junio de 2010 